\title{
An improved force controller with low and passive apparent impedance for series elastic actuators
}

\author{
Wolfgang F. Rampeltshammer, Arvid Q.L. Keemink, Herman van der Kooij
}

\begin{abstract}
This work presents a force controller for series elastic actuators that are used in gait robots such as exoskeletons, prostheses, and humanoid robots. Therefore, the controller needs to increase the bandwidth of the actuator, lower its apparent impedance for disturbance rejection or effortless interaction with a human user, and to stably interact with any (dynamic) environment. For gait, these environments are changing discontinuously, thus creating regular impacts. In this work, we propose the use of an inner loop PD controller to increase the bandwidth of the actuator, alongside an outer loop disturbance observer (DOB) to lower the apparent impedance of the actuator. To increase the controlled bandwidth of the actuator, we introduce a novel tuning method for the PD controller that allows for independent tuning of bandwidth and damping ratio of the controlled plant. The DOB, which is introduced to reject disturbances by lowering the apparent impedance, causes the apparent impedance to turn non-passive, resulting in potential contact and coupled instability of the actuator. To enable unconditionally stable interactions with any environment, we scale down the DOB contribution such that it lowers the apparent impedance while remaining passive. The proposed tuning method and DOB adaptation were evaluated on a test-setup by identifying the torque controller's transfer behavior and by identifying the apparent impedance of the actuator. The results of these tests showed that the proposed tuning method can separately tune bandwidth and damping ratio, while the DOB adaptation is able to trade-off the reduction of the apparent impedance with its passivity.
\end{abstract}

\section{INTRODUCTION}

Series elastic actuators (SEA) with their compliant properties are a popular choice for lower limb exoskeletons [1][4], humanoid robots [5], [6], and powered prostheses [7][10]. However, despite their regular use for robots involved in locomotion, the control of SEAs used for locomotion remains challenging. This is mostly due to the impact during heel strike, as well as the high torques required. In this work, we present the SEA torque controller that was developed for the Symbitron exoskeleton [11], a twelve degree of freedom exoskeleton for paraplegic and healthy users. It guarantees accurate torque tracking alongside a passive and low apparent actuator impedance.

For a SEA torque controller that is used in gait robots, several requirements were defined: first, the torque tracking bandwidth must be sufficiently high $(20-40 \mathrm{~Hz})$ : it should be

Manuscript received ..., 2019; ...

This work was supported by NWO under Grant 14429 (Corresponding author: W.F. Rampeltshammer)

The authors are with the Department of Biomechanical Engineering, University of Twente, Enschede, 7522NB, The Netherlands (e-mail: w.f.rampeltshammer@utwente.nl).

Color versions of one or more of the figures in this paper are available online at http://ieeexplore.ieee.org.

Digital Object Identifier ... at least $20 \mathrm{~Hz}$ for normal human gait, which has frequencies up to $10 \mathrm{~Hz}$, and even higher, if more immediate reactions, such as balance responses, are required (R0). Furthermore, the controller must be able to handle torques that support gait, i.e. at least $100 \mathrm{Nm}$ (R1). Third, the controlled apparent actuator impedance, i.e. the residual interaction force when a user or environment moves the force controlled joint, should be as low as possible to ensure transparency (R2). Fourth, the controller has to be stable in any contact situation, i.e. not generate oscillations during any environment interaction. Hence, the apparent actuator impedance should be passive (R3). Requirements R2 and R3 translate to user comfort and safety for exoskeletons or prostheses, because the actuator will have small resistance to user motions and will have good impact absorption. The latter also benefits humanoid robots during gait. Finally, the controller needs to work for a power limited system, such as an autonomous exoskeleton (R4). This implies practical limits on current and voltage.

Some existing controllers already achieve parts of those requirements. Hopkins et al. [6] implemented a disturbance observer (DOB) based controller for the THOR humanoid and demonstrated its effectiveness for gait. However, their controller introduced oscillations into the ankle joint. Paine et al. [12] proposed a different DOB approach for the Valkyrie humanoid, and successfully demonstrated its low impedance in human robot interaction for its arms. However, they did not use their controller for its legs [5]. The control approach for the iCub [13] uses a compliance regulation filter to track a desired position while rendering a specified impedance. This position control approach has been shown to work for impacts, which shows its applicability to walking. None of these methods could, however guarantee a passive apparent impedance.

For lower limb exoskeletons, Witte et al. [2] used PD based approaches alongside powerful offboard actuation for their ankle exoskeleton to deliver high torques. Their use of Bowden cable actuation for unidirectional motion guaranteed a low apparent impedance and also passivity. A similar approach was used for a prostheses emulator by Caputo et al. [9]. Other exoskeleton or prostheses approaches for SEA control mostly suffer from low bandwidth [1], [7], only demonstrate torque tracking for low torques in the range of $0.5-8 \mathrm{Nm}$ [3], [14], or give little information about the controller used [3], [4], [10]. Besides using feedback controllers, for prostheses, a viable option is also pure feedforward control [8]. However, this option is only feasible as long as the prostheses model is close enough to reality.

Another gait robot with SEAs, namely the LOPES gait trainer, was controlled by adopting a cascaded PID approach, 
and introduced tuning rules for passivity [15]. However, this approach also suffers from a limited torque bandwidth, and controls for motor velocity in the inner loop, making it impossible to use torque feedforward. Another promising approach was developed for the gait trainer SUBAR [14], which uses a DOB approach alongside a feedforward filter, to make it transparent to the user. However, its effectiveness was only demonstrated for low torques.

Extensive research into SEA control, tested for various purposes mostly on single actuators, also introduces a broad set of options. These approaches include position control with DOB [16], a torque-based DOB [17], cascaded PID controllers [18], [19], and admittance control [20]. The latter is mainly used to render high stiffness [21]. However, those approaches tend to become unstable when in contact with stiff environments [22], which is problematic during heel strike and ground contact. The mentioned DOB approaches have also been augmented by modeling the load side for the controller design [23] or compensating for the load side by applying acceleration feedback [24], [25]. The former suffers from the necessity to model the load side, while the latter is dependent on good acceleration measurements. Furthermore, good results were also achieved using adaptive controllers [26], [27], or controllers using iterative learning approaches [28]. However, their performance in uncertain or changing environments remains unclear.

In this paper, we present a novel plant shaping controller for SEAs that also renders a low and passive apparent impedance. The basic structure for the controller is the DOB controller described by Paine et al. [12] and Kong et al. [16] with an inner loop PD controller and an outer loop DOB. Compared to their work, the presented PD controller can be independently tuned for the desired damping ratio and bandwidth. This independent tuning method also results in low PD gains, making the approach suitable for autonomous systems. Furthermore, the outer loop DOB controller minimizes the apparent actuator impedance, while making the apparent impedance non-passive. To guarantee its passivity, an adaptation of the DOB is introduced that can reduce the phase lead of the apparent impedance, thus achieving unconditionally stable interactions with any environment. With the adapated DOB, we achieved a passive apparent impedance, however, at the same time, the apparent impedance of the actuator gets higher, and accordingly the torque tracking accuracy gets slightly worse. As a result the presented controller presents a trade-off between low apparent impedance and unconditional interaction stability.

The proposed control approach is verified by conducting system identifications of the torque transfer and the apparent actuator impedance on a test setup. The obtained results show that the proposed approach is able to independently tune bandwidth and damping ratio, and that the DOB adaptation achieves a passive apparent impedance.

Following the introduction, the proposed controller is described in section II. The controller is evaluated in section III and its performance discussed in section IV. The paper concludes with section V.

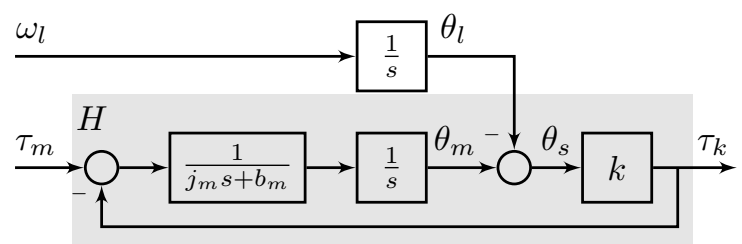

Fig. 1. Model of the SEA from motor torque $\tau_{m}$ to spring torque $\tau_{k}$. The load side dynamics are generalized to a general velocity input $\omega_{l}$

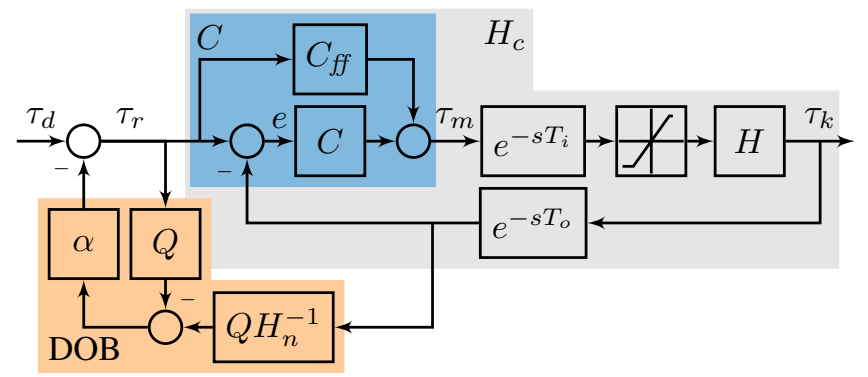

Fig. 2. Proposed torque controller for SEAs. For simplicity, the load side velocity input $\omega_{l}$ is omitted. The SEA $H$ with motor saturation and communication delays $T_{i}, T_{o}$ is controlled with an inner loop controller $C$ with additional torque feedforward $C_{f f}$. On the outer loop, a disturbance observer DOB with nominal model $H_{n}$, DOB filter $Q$, and DOB gain $\alpha$ shapes the apparent actuator impedance.

\section{CONTROL APPROACH}

Based on the requirements defined in Sec. I, a PD controller with DOB is introduced [17], [29]. For this controller, a novel tuning method is introduced that derives the correct bandwidth equation for a PD controlled SEA, and uses that to independently tune damping ratio and bandwidth of the plant. Additionally, we adapt the DOB such that the apparent impedance of the SEA to achieve unconditional contact stability.

\section{A. SEA model}

The model of a SEA can be seen in Fig. 1. The spring couples the interaction between motor and environment, which is generalized as an arbitrary load side velocity $\omega_{l}$, for the purposes of this work. This results in a spring torque $\tau_{k}$ of:

$$
\tau_{k}=\frac{k}{j_{m} s^{2}+b_{m} s+k}\left(\tau_{m}-\left(j_{m} s+b_{m}\right) \omega_{l}\right) .
$$

Here, $\tau_{m}$ denotes the motor torque, $j_{m}$ the effective motor side inertia, $b_{m}$ the effective motor side damping, and $k$ the stiffness of the series elastic element. This results in a torque transfer function for the system dynamics with a locked output $\left(\omega_{l}=0\right)$ :

$$
H(s)=\frac{\tau_{k}}{\tau_{m}}=\frac{k}{j_{m} s^{2}+b_{m} s+k},
$$

and the actuator's intrinsic impedance, or uncontrolled load disturbance sensitivity function:

$$
Z(s)=\frac{\tau_{k}}{-\omega_{l}}=\frac{k\left(j_{m} s+b_{m}\right)}{j_{m} s^{2}+b_{m} s+k},
$$

where $H$ denotes the plant torque transfer function, describing a SEA with locked output, and $Z$ the physical actuator 
impedance. For simplicity, it is assumed that the motor current controller of the plant is part of the plant model and that it has sufficiently high bandwidth compared to the achievable controlled bandwidth of a SEA. This plant $H$ is controlled by an inner loop controller $C$ with a feedforward term $C_{f f}$, as shown in Fig. 2. In the following, the feedforward term is set to $C_{f f}=1$, and the inner loop feedback controller is defined as a PD controller $C=K_{D} s+K_{P}$, with $K_{D}$ the differential (D) gain, and $K_{P}$ the proportional (P) gain. Alongside the communication delays from controller to motor $T_{i}$, and from torque sensor to controller $T_{o}$, as well as the complete delay $T_{d}=T_{i}+T_{o}$, this controller results in the controlled plant $H_{c}$ with a corresponding apparent actuator impedance without DOB, $Z_{c}$ :

$$
\begin{aligned}
& H_{c}(s)=\frac{\tau_{k}}{\tau_{r}}=\frac{k e^{-s T_{i}}\left(1+K_{P}+K_{D} s\right)}{j_{m} s^{2}+b_{m} s+k\left(1+e^{-s T_{d}}\left(K_{P}+K_{D} s\right)\right)}, \\
& Z_{c}(s)=\frac{\tau_{k}}{-\omega_{l}}=\frac{k\left(j_{m} s+b_{m}\right)}{j_{m} s^{2}+b_{m} s+k\left(1+e^{-s T_{d}}\left(K_{P}+K_{D} s\right)\right)} .
\end{aligned}
$$

This inner loop controller is augmented by a disturbance observer of the PD controlled plant $H_{c}$, as shown in Fig. 2. This observer consists of the locked nominal plant model $H_{n}$, alongside a filter $Q$. As the nominal model $H_{n}$, we use the actual model $H_{c}$, described in (4), with all time delays set to zero. The selection of the filter $Q$ is discussed later in this section.

To show the effects of the DOB on the torque controller transfer, the time delay is treated as an additive parameter error, thus relating the actual with the nominal model $H_{c}=$ $H_{n}\left(1+\Delta_{H}\right)$. Here, $\Delta_{H}$ is the additive torque transfer error that also encompasses all modeling and parameter estimation errors. Based on the control diagram in Fig. 2, this results in a torque controller transfer function for the DOB of:

$$
H_{D O B}(s)=\frac{\tau_{k}}{\tau_{d}}=H_{n} \frac{1+\Delta_{H}}{1+\alpha Q \Delta_{H}}
$$

that has a corresponding apparent impedance of:

$$
Z_{D O B}(s)=\frac{\tau_{k}}{-\omega_{l}}=(1-\alpha Q) Z_{n} \frac{1+\Delta_{Z}}{1+\alpha Q \Delta_{H}},
$$

where $\alpha$ denotes the DOB gain, and $\Delta_{Z}$ the impedance parameter error, which can be related to the torque model error. This overall controller results in a controlled spring torque of:

$$
\tau_{k}=H_{D O B} \tau_{d}+Z_{D O B} \omega_{l}
$$

From this relation and the transfer functions in (6) and (7) it can be seen that in case of no modeling errors and no time delay, i.e. $\Delta_{H}=0$ and $\Delta_{Z}=0$, the DOB does not affect the spring torque transfer from the desired torque $\tau_{d}$. It does, however, always affect the torque caused by motions of the load side or external disturbances $\omega_{l}$. Hence, the apparent impedance $Z_{D O B}$ is indeed a load disturbance sensitivity function, that demonstrates the controller's capability of rejecting external motion disturbances. As long as the DOB gain $\alpha$ is bigger than zero, the DOB will reject parts of the disturbances. In case of non-zero errors $\Delta_{H}$, the DOB also partially compensates these errors and thus also influences the transfer behavior from the desired torque.

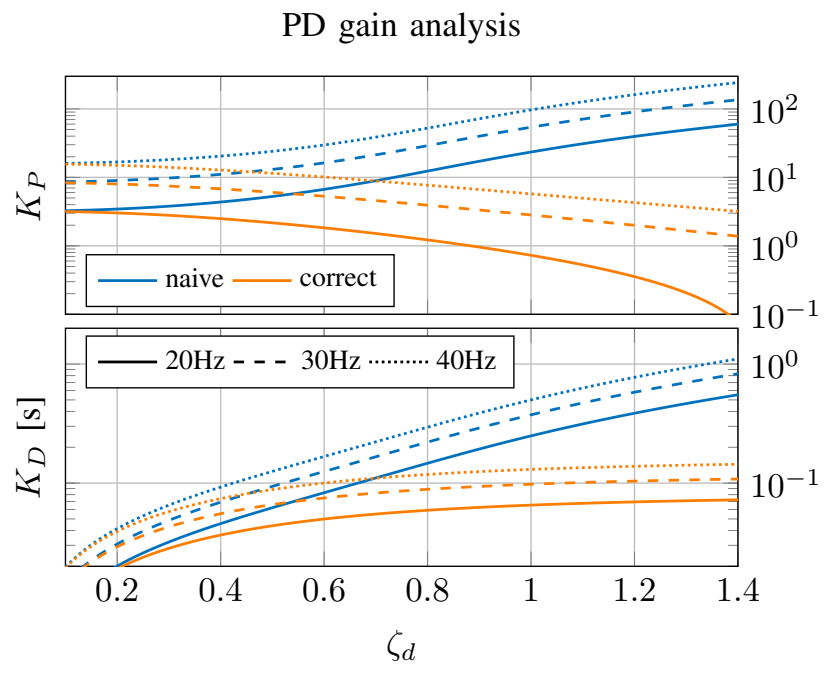

Fig. 3. Comparison between the naive (Eq. 9), and correct bandwidth equation (Eq. 13), and their effects on the PD gains $K_{P}$, and $K_{D}$. Using the correct bandwidth communication significantly reduces necessary controller gains. This observation is consistent for various desired bandwidths $\omega_{d}$.

\section{B. Naive controller tuning}

For the inner loop PD controller, several tuning methods have been described, such as optimization using the LQ method [16], manual tuning [17] or treating the system as a second order plant, and tuning its bandwidth and damping ratio accordingly [12]. We required the controller be usable for power limited systems (R4), making it necessary to reduce peaks in the torque control transfer, and to adapt the bandwidth of the system dynamically to the desired tracking signals (R0). Based on these requirements, we decided to adapt the method of tuning bandwidth and damping ratio, proposed by Paine et al. [12], and also used for a different controller by Losey et al. [27], for use of SEAs in locomotion robots.

Their approach uses the desired torque controller bandwidth $\omega_{d}$, and damping ratio $\zeta_{d}$ to derive the proportional $K_{P}$ and differential gain $K_{D}$. Therefore, this method approximates the controlled plant as a pure second order system without a zero in the transfer numerator. The effects of this approximation are outlined in the following. For the tuning of the PD gains, all time delays are assumed to be zero. Using the definition of the bandwidth of a second order system without zeros in the numerator:

$$
\omega_{d}=\omega_{c} \sqrt{1-2 \zeta_{d}^{2}+\sqrt{1+\left(2 \zeta_{d}^{2}-1\right)^{2}}}
$$

where $\omega_{c}$ is the natural frequency of the controlled system, the following tuning rules were derived:

$$
\begin{gathered}
K_{P}=\frac{\omega_{c}^{2} j_{m}}{k}-1, \\
K_{D}=\frac{2 \zeta_{d} \sqrt{j_{m} k\left(1+K_{P}\right)}-b_{m}}{k} .
\end{gathered}
$$

The resulting gains for various design parameters are shown in Fig. 3 as the "naive" method (as implied by Paine et al. [12]), and the resulting closed loop transfer function in Fig. 4. These figures show that this method does not allow for 


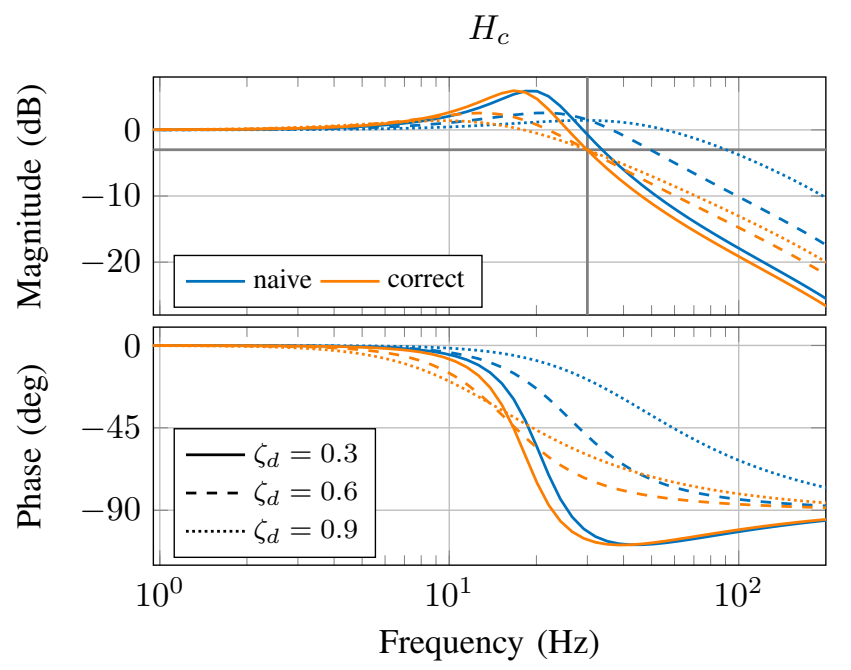

Fig. 4. Bode plots of the controlled plant $H_{c}$ using the "naive", and correct bandwidth equation. All controllers were tuned for a desired bandwidth $\omega_{d}$ of $30 \mathrm{~Hz}$. The bandwidth increasing effect of the naive approach is shown, while the correct equation achieves the desired bandwidth (-3dB line) for each $\zeta_{d}$.

decoupled tuning of bandwidth and damping ratio: increasing the damping ratio automatically increases the bandwidth. This increase of bandwidth can only be avoided if the $\mathrm{D}$ part of the controller is replaced with absolute damping feedback [27].

In this case, however, the undesired increase of bandwidth limits the method's applicability for a power limited system (R4), considering a possible bandwidth increase of up to $70 \mathrm{~Hz}$. This effect is caused by approximating the PD controlled plant as a pure mass spring damper system that ignores the zero in the transfer function of Eq. 4. Therefore, it is necessary to manually decrease the desired bandwidth such that the actual bandwidth approaches the desired one. This effect is more pronounced for an actuator with a very low motor side damping, as it is the case for our actuators.

\section{Proper tuning, decoupling damping ratio and bandwidth}

To overcome these limitations, we generalized the bandwidth equation for such second order systems to second order systems with an additional zero, i.e. the correct transfer of PD controlled SEAs. This generalization allows for a decoupled design of bandwidth and damping ratio of the torque controller by utilizing the bandwidth increasing effect of the system zero. Therefore, we computed the bandwidth of the controlled plant $H_{c}$ based on the $-3 \mathrm{~dB}$ crossing, i.e. half the power of the original signal:

$$
\left|H_{c}\left(j \omega_{d}\right)\right| \triangleq-3 \mathrm{~dB}=\frac{1}{\sqrt{2}} .
$$

Inserting the system expression, and solving the equation for the bandwidth $\omega_{d}$ results in the generalized bandwidth equation:

$$
\omega_{d}=\omega_{c} \sqrt{1-2 \zeta_{d}^{2}\left(1-2 \delta_{\zeta}^{2}\right)+\sqrt{1+\left(2 \zeta_{d}^{2}\left(1-2 \delta_{\zeta}^{2}\right)-1\right)^{2}}}
$$

Theoretical apparent impedance

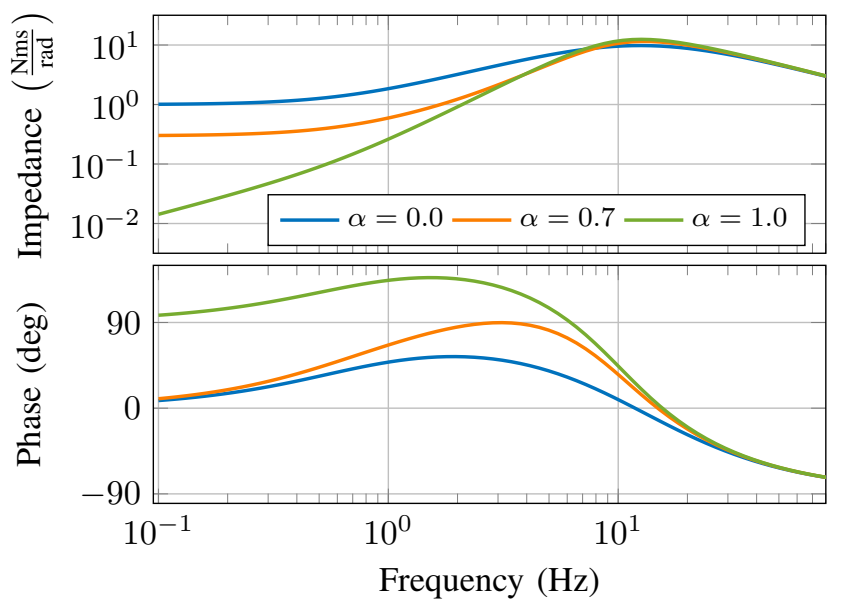

Fig. 5. Bode plots of the actuator impedance $Z_{D O B}$. The corresponding controller was tuned with the parametrization $\left(\omega_{d}, \zeta_{d}, \omega_{q}\right)=(30 \mathrm{~Hz}, 1,10 \mathrm{~Hz})$. The apparent impedance for various DOB gains is shown: DOB off $(\alpha=0)$, passive DOB $(\alpha=0.702)$, and full DOB $(\alpha=1)$. The effects of the DOB gain can be observed: lowering the gain reduces the phase lead, while increasing the apparent impedance. The passive condition still shows a lower impedance than without the DOB, demonstrating the usefulness of an adapted DOB.

with a damping generalization factor:

$$
\delta_{\zeta}=1-\frac{\zeta_{n} \omega_{n}}{\zeta_{d} \omega_{c}}=1-\frac{b_{m}}{b_{m}+k K_{D}},
$$

in which $\zeta_{n}$ and $\omega_{n}$ are the undamped natural frequency and damping of the uncontrolled plant, derived from the unactuated plant model in Eq. 2. The complete derivation can be found in Appendix A. Using the presented generalization, an implicit method can be used to find the correct PD gains.

The damping generalization factor in Eq. 14 can be interpreted as the amount of relative damping increase of the controlled plant. The larger the difference between $b_{m}$, and $b_{m}+k K_{D}$, the higher the generalization factor. The generalized bandwidth Eq. 13 has the equation for a pure second order system as in Eq. 9 as its limit case if $\delta_{\zeta}$ goes to zero. Hence, it can be seen that the tuning method proposed by Paine et al. [12] is a special case, which only works for PD controllers, if the desired damping is similar to the actual damping.

The decoupling effects of the adapted bandwidth equation are shown in Fig. 4. The figure shows that the bandwidth stays constant, even if the damping ratio is increased, thus satisfying requirement (R0). Additionally, the PD gains are far lower compared to the naive approach, as shown in 3. As a result, this low gain approach also satisfies the power limitation requirement $(\mathrm{R} 4)$.

\section{Reducing apparent impedance with disturbance observer}

The presented PD controller is on its own sufficient to fulfill requirements R0, R1 and R4 with low gains as well as guaranteeing a tunable bandwidth. However, the actuator's apparent impedance for the presented controller is still relatively high, as shown in Fig. 5 for $\alpha=0$. To satisfy requirement $\mathrm{R} 2$, the controlled apparent impedance has to be lowered. 
This is identical to improving the disturbance rejection of the controller. Therefore, an outer loop disturbance observer, as described in Eq. 6, is introduced. Besides rejecting external disturbances and thus lowering the apparent impedance, the DOB also helps to eliminate unmodeled effects such as static friction [14], i.e. steady state errors, or to decouple joints [12]. This disturbance rejection further helps to decrease the necessary power, by feed-forwarding unmodeled disturbances, which reduces the control error. Therefore, the DOB also contributes to requirement R4 by lowering the power necessary for control.

With an outer loop DOB, three additional tuning options are introduced: the nominal model $H_{n}$, the DOB filter $Q$, and the DOB gain $\alpha$. The main purpose of the DOB is to reduce the actuator impedance. Hence, the nominal model is selected to be as close as possible to the actual closed loop dynamics [30], expressed in (4), to reject disturbances, instead of shaping plant behavior with the nominal model in the DOB. Hence, apparent impedance and torque tracking behavior can be tuned independently. The relative degree of the filter $Q$ has to be equal to, or higher than, the relative degree of the plant [29], to make the model inversion proper. For the lowest possible impedance and phase lead, the relative degree of the filter should also be minimal. However, a first order filter is not feasible for implementation due to the transfer function of $Q H_{n}^{-1}$ having a degree of 0 , thus not making it strictly proper. Therefore, a second order Butterworth filter was selected for the DOB filter:

$$
Q(s)=\frac{\omega_{q}^{2}}{s^{2}+\sqrt{2} \omega_{q} s+\omega_{q}^{2}},
$$

where $\omega_{q}$ is the cutoff frequency of the filter. Instead of using a Butterworth filter, the filter could also be specifically designed to shape the DOB behavior, as outlined by Schrijver et al. [29]. As seen in Fig. 5, the introduction of a DOB reduces the apparent impedance, but at the same time introduces a phase lead to the impedance transfer. As a result, the apparent impedance is no longer passive, violating requirement R3, and possibly causing contact and coupled instability.

\section{E. Impedance passivity of disturbance observers}

To make the controlled apparent impedance passive again, the DOB gain $\alpha$ is introduced: by lowering it, the maximum phase lead of the controlled apparent impedance is reduced such that the apparent impedance becomes passive. To avoid positive feedback, the DOB gain should always be positive, i.e. $\alpha \geq 0$.

This gain has to be introduced, because the naive DOB controlled system impedance is not passive, even if exact system knowledge is assumed, as shown in Fig. 5. This effect is especially prominent for actuators with a low ratio of motor damping $b_{m}$ to motor inertia $j_{m}$, which implies a low mechanical time constant. Furthermore, it depends on the desired damping ratio and bandwidth of the controller, as is shown in Fig. 6. To guarantee a passive apparent actuator impedance in those cases, while increasing the system bandwidth, the DOB gain has to stay below 1 . As a result, instead of behaving like

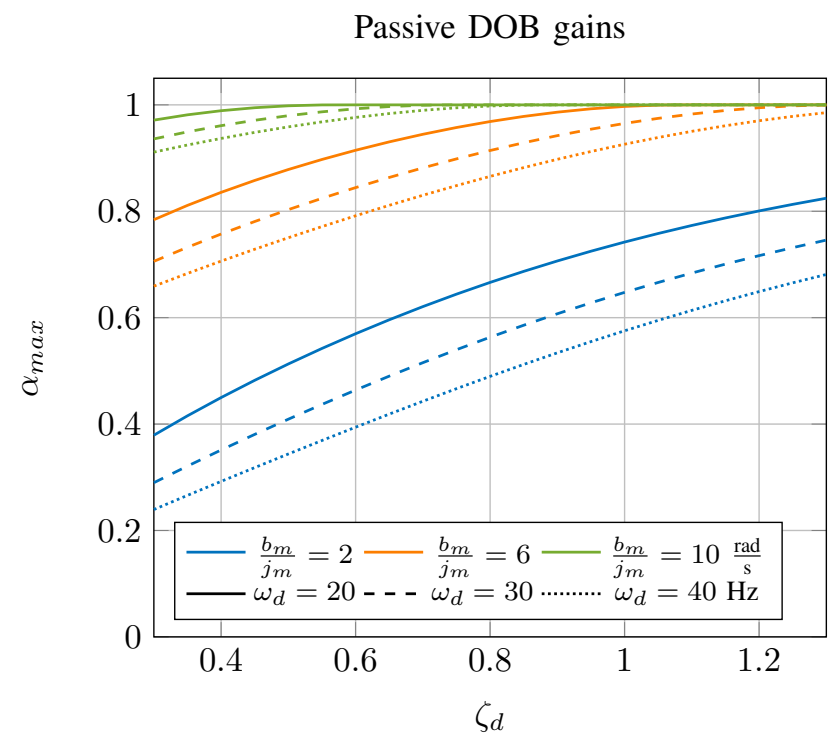

Fig. 6. Maximum admissible DOB gains $\alpha_{\max }$ that guarantee a passive impedance are evaluated for various ratios of motor inertia to damping $\frac{j_{m}}{b_{m}}$, various desired bandwidths $\omega_{d}$ and damping ratios $\zeta_{d}$. It is shown that $\alpha_{\max }$ is mainly dependent on the physical properties of the motor, and less by tuning parameters.

a feedback integrator, the DOB behaves like a leaky feedback integrator.

Reducing the DOB gain results is a trade-off between torque tracking performance as well as good disturbance rejection and unconditional interaction stability. It increases the apparent impedance at low frequencies, and affects the torque tracking performance in cases of imperfect model knowledge. As a result external disturbances cause higher torque tracking errors, compared to a DOB gain of one.

For passivity of the controlled apparent impedance, an upper bound for the DOB gain $\alpha$ can be found by utilizing the positive real condition for the actuator impedance:

$$
\operatorname{Re}(Z) \geq 0, \quad \forall \omega \in \mathbb{R}
$$

By solving this inequality, an upper bound for the DOB gain $\alpha_{\max }$ can be found that guarantees a passive actuator impedance. The exact condition can be found in Appendix B. As shown in Fig. 6, the maximum gain is mostly influenced by the reflected physical motor parameters $j_{m}$ and $b_{m}$, and the desired damping ratio $\zeta_{d}$, but less by the desired bandwidth $\omega_{d}$. The conclusion is that for real systems there is the possibility to set $\alpha$ not much lower than 1.0, to achieve better residual/apparent impedance and also disturbance rejection by the DOB.

\section{F. Controller framework}

Based on these insights, a controller for an arbitrary actuator can be designed as follows: first, select a desired damping ratio $\zeta_{d}$, and bandwidth $\omega_{d}$. Then use the implicit equations (13), (10) and (11) to calculate the PD gains. Next decide on an appropriate cut-off frequency for the DOB filter $Q$, and determine the DOB gain $\alpha_{\max }$ with the implicit equations (29) in Appendix B. The resulting controller achieves the desired 


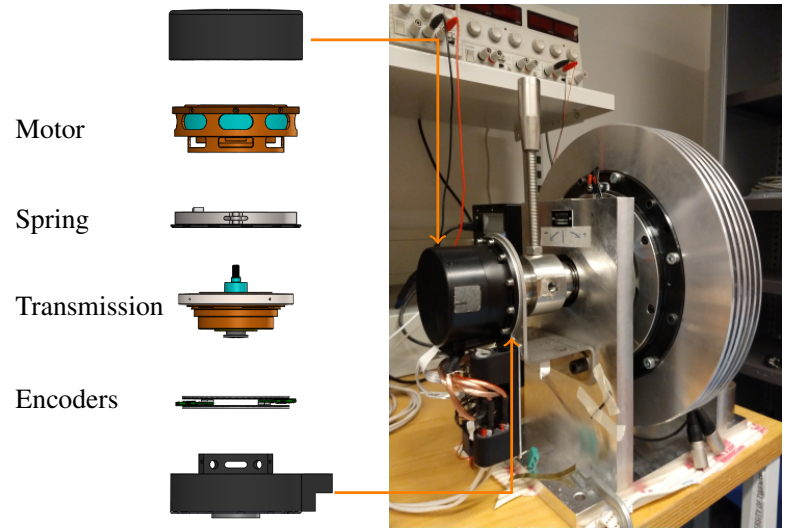

(a) CAD model

(b) Actuator setup

Fig. 7. CAD model of the actuator and setup of the actuator. CAD model depicts motor, harmonic drive, series elastic element and encoders. Actuator setup showing the actuator, its movable output side, as well as its attachment to a magnetic brake for locked output tests.

TABLE I

SEA SPECIFICATIONS

\begin{tabular}{lc}
\hline \hline Weight & $1.55 \mathrm{~kg}$ \\
Gear ratio (speed reduction) & $1: 100$ \\
Maximum speed & $5 \mathrm{rad} / \mathrm{s}$ \\
Continuous motor torque & $102 \mathrm{Nm}$ \\
Maximum motor torque & $225 \mathrm{Nm}$ \\
Spring stiffness $k$ & $1534 \mathrm{Nm} / \mathrm{rad}$ \\
Operating voltage & $44 \mathrm{~V}$ \\
Reflected motor inertia $j_{m}$ & $0.9851 \mathrm{kgm}$ \\
Reflected motor damping $b_{m}$ & $1.9702 \mathrm{Nms} / \mathrm{rad}$ \\
\hline \hline
\end{tabular}

bandwidth and damping ratio, and has a passive apparent impedance.

\section{EXPERIMENTAL EVALUATION}

To evaluate the proposed controller framework two experiments, one for torque tracking, and one for minimal impedance, were conducted on a SEA in a test environment. In the following, we will first describe the actuator, followed by a detailed description of the experiments, and finally, their results.

\section{A. SEA test-setup}

The controller presented in this work is designed for the SEA of the Symbitron exoskeleton [11] and tested on one of its actuators as shown in Figs. 7a and 7b, with its specifications shown in Table I. A Tiger Motor U8-10(Pro), T-Motor, Nancheng, China, is reduced by a LCSG20 harmonic drive, Leader Drive, Jiangsu, China, which connects to a custom rotary spring. The motor is controlled by an iPOS8020-BX drive, Technosoft S.A., Switzerland, and communicates over EtherCAT. Furthermore, the actuator is equipped with two Aksim encoders from RLS (Renishaw), each with a resolution of 20 bits; one to measure the joint angle, and the other to measure the spring deflection. The motor position is measured by a MHM encoder, IC Haus, with a resolution of 16 bits. The encoders are attached to a custom sensor slave, running at $1 \mathrm{kHz}$ that also communicates over EtherCAT. The actuators are connected to a computer and interfaced using TwinCAT 3, Beckhoff Automation.

The load side of the actuator was designed to deliver torques up to $100 \mathrm{Nm}$, and can achieve speeds up to $5 \mathrm{rad} / \mathrm{s}$. In its current setup, time delays were determined to be $T_{i}=1 \mathrm{~ms}$, and $T_{o}=2 \mathrm{~ms}$. The reflected motor inertia was computed from the known motor inertia and gearbox specifications, and the reflected motor damping was identified from the open loop torque response of the actuator. For the experiment, the actuator was equipped with a handle on the output side to move it manually, and set up such that its output could also be locked, as shown in Fig. 7b.

\section{B. Experimental evaluation}

First, the torque transfer behavior of an actuator with locked output is identified for the combination of four different bandwidths $\omega_{d} \in\{20,30,40,50\} \mathrm{Hz}$, and five different damping ratios $\zeta_{d} \in\{0.7,0.9,1.1,1.3,1.5\}$ to evaluate the effectiveness of the proposed PD tuning method. The only omitted condition is $\left(\omega_{d}=20 \mathrm{~Hz}, \zeta_{d}=1.5\right)$ due to a required negative $K_{p}$. For all tested conditions, the DOB parameters were set to $\left(\omega_{q}=10 \mathrm{~Hz}, \alpha \in[0.58,0.96]\right)$. The filter bandwidth is kept the same to keep the controllers comparable, and the DOB gains are selected to guarantee a passive apparent impedance, i.e. $\alpha \leq \alpha_{\max }$. This is necessary, because the actuator can, due to the series elastic element, still move in a locked output condition, and consequently can get unstable if the apparent impedance is non-passive. The tested damping ratios were selected to reflect the desired range for implementation in gait: lower damping ratios for the ankles $\left(\zeta_{d}=0.9\right)$ to achieve faster torque changes during ground contact transitions, and higher ones $\left(\zeta_{d}=1.1\right)$ for the hip to achieve interaction torques with less overshoot, while bandwidths were selected to demonstrate the possible increase in bandwidth, which is over five times the uncontrolled bandwidth.

For the identification, the actuator is excited with 31 frequencies spaced uniformly on a linear scale between 0.1 and $60 \mathrm{~Hz}$, with amplitudes of $\tau_{d}=10 \mathrm{Nm}$ for $\omega_{i} \in[0.1,10]$ $\mathrm{Hz}, \tau_{d}=7.5 \mathrm{Nm}$ for $\left.\left.\omega_{i} \in\right] 10,15\right] \mathrm{Hz}, \tau_{d}=5 \mathrm{Nm}$ for $\left.\left.\omega_{i} \in\right] 15,20\right] \mathrm{Hz}$, and $\tau_{d}=2 \mathrm{Nm}$ for $\left.\left.\omega_{i} \in\right] 20,60\right] \mathrm{Hz}$. Each frequency was presented separately, and for ten periods and averaged in the frequency domain for the computation of the torque transfer. The amplitudes were designed to prevent motor current saturation for any of the evaluated conditions. For processing, the first full period was discarded to eliminate transient effects, and the last quarter period of the excitation signal was discarded to eliminate the effects of stopping the torque profile.

Second, we evaluate the feasibility of the proposed controller for high torques. Therefore, the actuator output is locked, and a torque step of $\tau_{d}=70 \mathrm{Nm}$ is applied to the following controller parametrizations: $\left(\omega_{d} \in\{30,40\} \mathrm{Hz}, \zeta_{d} \in\{0.7,1.1\}, \omega_{q}=10 \mathrm{~Hz}, \alpha=0.7\right)$.

The final value of the step was selected as a trade-off between high torques, and to protect the actuator, especially the gearbox, from damage. The conditions were selected to 
demonstrate that the proposed controller can, at high torques, reduce the overshoot of the controlled system by increasing the damping ratio, and that different bandwidths have a similar overshoot.

Third, the apparent impedance of the actuator is identified for three different DOB gains $(\alpha \in\{0.0,0.7,0.9\})$ to evaluate its effect on the phase lead and minimal impedance of the system. The rest of the controller is tuned as follows: $\left(\omega_{d}, \zeta_{d}, \omega_{q}\right)=(30 \mathrm{~Hz}, 0.7,10 \mathrm{~Hz})$. To identify the apparent impedance, the actuator output is manually moved at various frequencies, up to around $10 \mathrm{~Hz}$. The excitation amplitude at low frequencies $\left(\omega_{i}=0.1 \mathrm{~Hz}\right)$ is around $\omega_{l}=0.5 \mathrm{rad} / \mathrm{s}$. For all other conditions the excitation amplitude is between $\omega_{l}=1$ and $\omega_{l}=2 \mathrm{rad} / \mathrm{s}$. Unfortunately it is impossible to get the amplitudes more consistent with manual excitation. By using a metronome, we attempted to ensure the consistency of excited frequencies across conditions.

Additionally, we conducted an impact experiment with a controller with passive and another one with non-passive apparent impedance to highlight the importance of apparent impedance passivity. The behavior of the actuator can be seen in a video, which can be found in the supplementary material to this paper. For this experiment, no data is presented, because all relevant information is already shown with the identification of the apparent impedance.

For both identification experiments, the data is transformed to the frequency domain, and Bode diagrams for both the impedance and torque transfer functions are generated, as shown in Figs. 8 and 9. For the step response tests, the response is shown in time domain, as shown in Fig. 10

\section{Results}

The impedance identification experiments, shown in Fig. 8, demonstrate that the DOB reduces the apparent impedance of the actuator thus effectively rejecting external disturbances. Furthermore, the experiment also demonstrates that a DOB does not necessarily have a passive impedance, as shown by the condition $\alpha=0.9$. As it can be seen for the condition $\alpha=0.9$, the phase crosses $90^{\circ}$, and as such, the apparent impedance is not passive, in contrast to the controller with a deactivated DOB $(\alpha=0)$. Therefore, decreasing the DOB gain decreases the maximum phase lead, which makes a passive apparent impedance possible. The condition $\alpha=0.7$ should have theoretically been passive, however, due to modeling uncertainties, its phase is slightly above 90 degrees. For the impedance magnitude, the tradeoff between passive impedance and low apparent impedance at low frequencies can be seen: decreasing the DOB gain increases the apparent impedance. The magnitude difference between measured and modeled data at low frequencies is probably caused by position dependent friction in the harmonic drive which is not compensated for, but affects the measured impedance at those very low frequencies with correspondingly low velocities.

The results of the torque tracking experiments, shown in Fig. 9, demonstrate that the bandwidth of the actual system is approximately equal to the desired bandwidth. The same
Measured apparent impedance

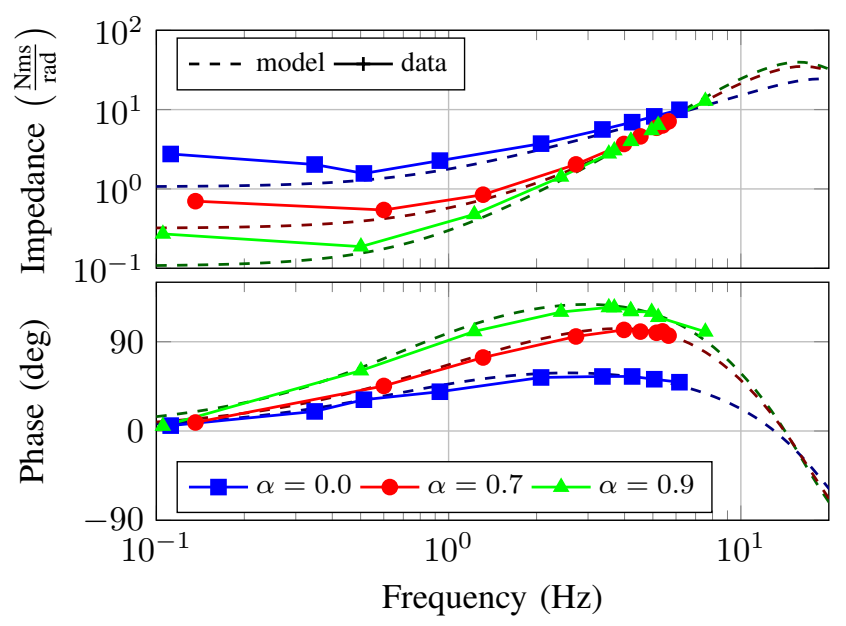

Fig. 8. Evaluating the effectiveness of the DOB gain on impedance passivity while applying no torque, i.e. $\tau_{d}=0$ : By manually exciting the actuator at various frequencies, the apparent impedance is identified for three DOB conditions $\alpha \in\{0,0.7,0.9\}$. The identified controller is tuned as follows: $\left(\omega_{d}, \zeta_{d}, \omega_{q}\right)=(30 \mathrm{~Hz}, 0.7,10 \mathrm{~Hz})$. The interpolated excited data points as well as the corresponding modeled impedance are shown. The theoretical effect of lowering DOB gains, i.e. less phase lead, and higher impedance can be observed. Furthermore, the benefit of using and adapted DOB is demonstrated by its reduced impedance compared to the no DOB condition.

holds for the tested damping ratios: lower damping ratios increase the peak, while the peaks are consistent over different bandwidths. For conditions with $\zeta_{d}=1.3$ and $\zeta_{d}=1.5$, the observable difference is negligible. Hence, it can be seen that there is a limit to introducing virtual damping to the controlled plant. Observed differences in peak heights and between desired and actual bandwidths can be attributed to model mismatches. In the $\omega_{d}=20 \mathrm{~Hz}$ condition, the DOB filter still allows for a compensation around the desired bandwidth. For higher desired bandwidths, this is no longer possible, resulting in the observed bandwidth mismatches. The observable phase drop at around $10 \mathrm{~Hz}$ is caused by communication delays, and the implemented filtered derivative for the PD controller. The special case $\left(\omega_{d}=50 \mathrm{~Hz}, \zeta_{d}=0.7, \alpha=0.7\right)$ of a controller with a non-passive apparent impedance demonstrates the importance of impedance passivity: At a frequency of 20 $\mathrm{Hz}$, the phase of the non-passive condition jumps, because the actuator excites the eigenfrequency of the table of the experimental setup. Based on the mounting table's resonance, and the not perfectly locked output of the actuator, a strong phase drop, and corresponding resonance peak is observable. In the passive condition $\left(\omega_{d}=50 \mathrm{~Hz}, \zeta_{d}=0.7, \alpha=0.58\right)$, this resonance peak caused the experimental table cannot be observed anymore. This effectively demonstrates the importance of a passive apparent impedance for accurate torque tracking.

The step experiments, shown in Fig 10, demonstrate that the controller still works when used with high desired torques. The results from this experiment confirm the results from the system identification experiments: lower desired damping ratios increase the overshoot and that damping ratios behave similarly across desired bandwidths. The effect of a higher 


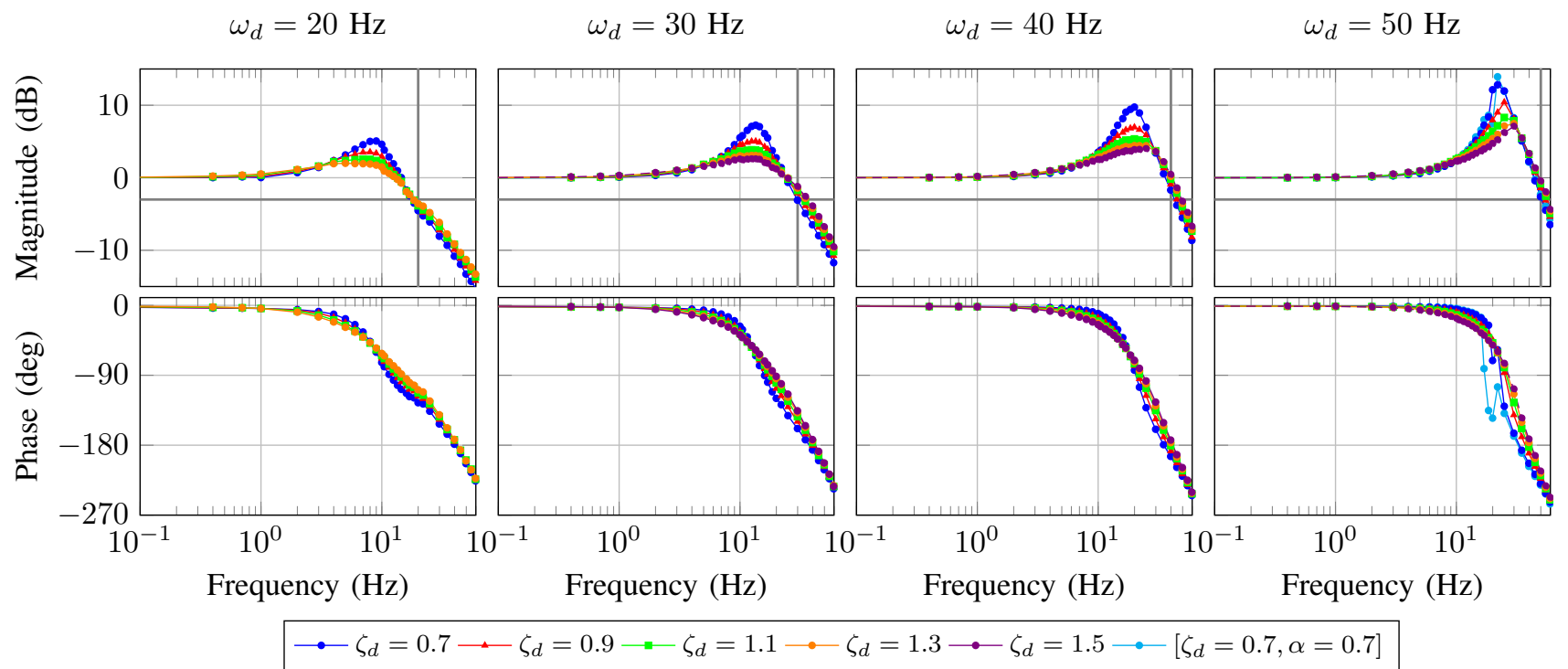

Fig. 9. Systematic system analysis of various controller parametrizations. Desired bandwidth is stated in the title, and the desired damping ratios are stated in the legend. DOB was tuned identically for all conditions: $\omega_{q}=10 \mathrm{~Hz}$, and $\alpha=0.7$. The system output was locked for all experiments, and the system was excited with a sine wave per target frequency ( 31 frequencies ranging from $0.1 \mathrm{~Hz}$ to $60 \mathrm{~Hz}$ ), for ten periods. The damping ratio of $\zeta_{d}=1.5$ was omitted for the bandwidth of $\omega_{d}=20 \mathrm{~Hz}$ due to a negative P gain. It can be seen that the actual bandwidth is consistently near the desired bandwidth, while a decrease in damping ratio consistently decreases the resonance peak, up to a damping ratio of $\zeta_{d}=1.3$. Differences in bandwidth, are most likely caused by time delays, and model mismatch. The phase drop at $10 \mathrm{~Hz}$ is a result of time delay and the implemented derivative filter. The spikes in the phase transfer of condition $\left(\omega_{d}=50, \zeta_{d}=0.7, \alpha=0.7\right)$ are caused by a non-passive apparent impedance which allowed resonance with the experimental table.

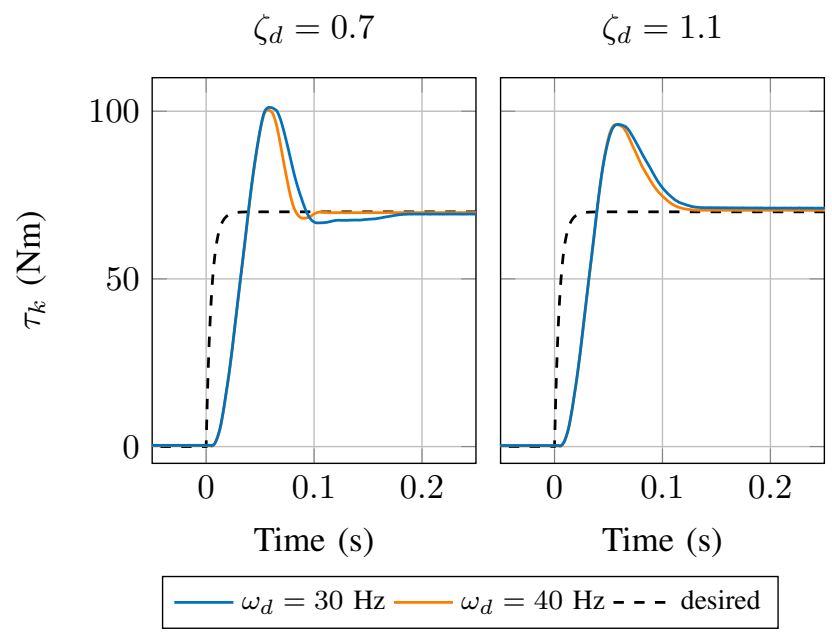

Fig. 10. Mean of 3 repeated step responses for a low pass filtered step $\tau_{d}=$ $70 \mathrm{Nm}$, tested for four different controller parametrizations: $\omega_{d} \in\{30,40\}$ $\mathrm{Hz}, \zeta_{d} \in\{0.7,1.1\}$. Damping ratios are shown in different figures, bandwidth differences are marked in different colors. It can be seen that all controllers behave identically during rise time. This is caused by a saturating motor current. However, the overshoot is still less for a higher damping ratio, and that a higher bandwidth is settling slightly faster. Overshoot for the respective damping ratios are similar.

bandwidth can be seen with a reduced settling time for the higher bandwidth. For the rising edge of the step, and accordingly the actual torque, there is no difference between conditions, because the motor current saturates for all conditions, due to the high instantaneous error. Another interesting point is that the actual torque for condition $\left(\omega_{d}=30 \mathrm{~Hz}, \zeta_{d}=1.1\right)$ does not settle at the desired torque $\tau_{d}=70 \mathrm{Nm}$. This is caused by the relatively low $\mathrm{P}$ gain, which in this case is not sufficient to reduce the torque error to zero while friction is present.

\section{DISCUSSION}

As described in the results section, the proposed control approach can shape the bandwidth and damping ratio independently. Furthermore, the proposed DOB gain allows for a passive apparent impedance. This improves the process of tuning the controller on the actual hardware and also allows additional adjustments based on various actuator configurations. Especially for exoskeletons and prostheses, the option to make the impedance passive helps with the trade-off between minimal apparent impedance, and contact stability.

However, the proposed approach can result in decreased performance for some controller parametrizations. At high damping ratios, the $\mathrm{P}$ gain can become low enough to negatively affect torque tracking at low frequencies, as shown in Fig. 10 for $\zeta_{d}=1.1$ at a desired bandwidth $\omega_{d}=30 \mathrm{~Hz}$. This can also be somewhat seen in the torque identification for higher damping ratios of the condition $\omega_{d}=20 \mathrm{~Hz}$, where the magnitude response gets slightly lower as damping ratios increase, as shown in Fig. 9. Furthermore, the corresponding increase of the D gain for higher damping ratios, alongside the implemented derivative filter, most likely causes the small increase of bandwidth for increasing damping ratios, as seen for almost all conditions of the system identification. The only exception is the condition $\omega_{d}=20 \mathrm{~Hz}$, where the DOB still has enough influence to shape the plant at the desired bandwidth. However, an according increase of the DOB filter bandwidth $\omega_{q}$ for higher desired bandwidths can easily introduce instabilities, as described by Schrijver et al. [29]. Another problem, especially for underdamped actuators, i.e. low ratios 
of $b_{m}$ to $j_{m}$, and as a result low natural damping ratios $\zeta_{n}$, is the negligible difference between conditions $\zeta_{d}=1.3$, and $\zeta_{d}=1.5$ : This clearly demonstrates that there is a physical limit to achievable damping ratios of the controlled torque transfer. Hence, it should be impossible to completely eliminate the peak of a SEA that is controlled as proposed. A solution for the overshoot can be the method proposed by Losey et al. [27], which eliminates the resonance peak by introducing absolute damping feedback. However such an approach comes at the cost of a non-passive torque transfer, as well as a far higher apparent impedance. Furthermore, the impedance passivity of the proposed controller is a problem: As seen by condition $\left(\omega_{d}=50 \mathrm{~Hz}, \zeta_{d}=0.7\right)$, a non-passive impedance, easily causes resonances of the actuator with the environment, negatively affecting torque tracking accuracy. Apparent impedance passivity, is achievable for the proposed control approach, making a trade-off between minimizing the apparent impedance as well as accurate torque tracking and contact stability possible and necessary. However, this tradeoff also demonstrates that there is a physical limitation of the proposed SEA controller: if the controller should be able to stably interact with any environment, the achievable minimum apparent impedance is limited. For exoskeletons, this will make the actuator have more resistance, as such reducing its comfort. Further research into impedance passivity of DOB based controllers is necessary to identify whether the presented limitation is physical, or if it can be eliminated by more sophisticated compensation mechanisms, such as designing the DOB filter $Q$ accordingly.

In conclusion, the presented control approach separates tuning of damping ratio and bandwidth. Furthermore, it has been demonstrated that a passive actuator impedance is achievable with a simple adaptation of the DOB. For the proposed control approach, the step response experiments, as shown in Fig. 10 have been shown that the controller can increase the plants bandwidth (R0), works for high torques (R1). The impedance identification, as shown in Fig. 8, has shown that it is possible to reduce the apparent impedance (R2), while keeping it passive (R3). Lastly, it has been shown that the proposed approach is useful for power limited systems (R4), because it possible to reduce the resonance peak, while designing the controller for a necessary bandwidth, as shown with the system identification experiment in Fig. 9.

Alongside the fulfilled requirements, the proposed control framework, also patched the two main weaknesses of the controller proposed by Paine et al. [12]: the coupling between damping ratio and bandwidth, and the non-passive apparent impedance. As shown with the experimental results, the final control framework is now easy to tune and implement, and can at the same time guarantee unconditional contact stability.

\section{CONCLuSion}

In this work, we developed a novel tuning approach for a PD controller of a SEA. This approach allows for the decoupled tuning of the damping ratio and bandwidth of the controlled actuator. Furthermore, we proposed a modification of the DOB approach that can achieve a passive apparent actuator impedance, resulting in unconditionally stable and oscillation free interaction with humans and environments. Both controller improvements have been confirmed experimentally on an actuator setup. The proposed requirements for a lowlevel controller for SEA-actuated gait robots were achieved.

Our follow-up efforts focus on implementing and testing the controller in a lower limb exoskeleton that has multiple degrees of freedom, to investigate if the proposed unconditional contact stability and the dynamic decoupling, as described by Paine et al. [17], holds for such an exoskeleton. In future work, we will also adapt the controller for low load side inertia, and test its robustness with respect to incorrect parameter estimates. Furthermore, the effects of time delay and derivative filtering on the system bandwidth will be investigated to improve the accuracy of the feedback gains and maximal DOB gain.

\section{ACKNOWLEDGMENTS}

This research is supported by the Netherlands Organisation for Scientific Research (NWO), project no. 14429. The authors also want to thank Gijs van Oort for productive discussions on the controller's performance during its development, and Niek Beckers for his valuable advice for the system identification.

\section{APPENDIX A \\ DERIVATION OF CORRECT BANDWIDTH}

To define the bandwidth of the system, the $-3 \mathrm{~dB}$ crossover frequency for the controlled plant $H_{c}$ without time-delay, i.e. $T_{d}=0$, has to be found.

$$
\left|H_{c}\left(j \omega_{d}\right)\right|=\frac{1}{\sqrt{2}}\left|H_{c}(j 0)\right|
$$

Defining:

$$
\begin{aligned}
R & =\frac{1}{\sqrt{2}}\left|H_{c}(j 0)\right|=\frac{1}{\sqrt{2}} \\
L & =\left|H_{c}\left(j \omega_{d}\right)\right| \\
& =\left|\frac{j k K_{D} \omega_{d}+k\left(1+K_{P}\right)}{-j_{m} \omega_{d}^{2}+j\left(b_{m}+k K_{D}\right) \omega_{d}+k\left(1+K_{P}\right)}\right|
\end{aligned}
$$

as the right and left hand side of Eq. 17 and inserting the definition from Eq. 10, and a reformulated Eq. 11 we obtain:

$$
\begin{aligned}
\frac{k K_{D}}{j_{m}} & =2 \zeta_{d} \sqrt{\frac{k\left(1+K_{P}\right)}{j_{m}}}-\frac{b_{m}}{j_{m}} \\
& =2 \zeta_{d} \omega_{c}-2 \zeta_{n} \omega_{n}=2 \zeta_{d} \omega_{c} \delta_{\zeta}
\end{aligned}
$$

with $\delta_{\zeta}=1-\frac{\zeta_{n} \omega_{n}}{\zeta_{d} \omega_{c}}$ results in

$$
\begin{aligned}
L & =\left|\frac{\omega_{c}^{2}+j 2 \zeta_{d} \omega_{c} \omega_{d} \delta_{\zeta}}{\omega_{c}^{2}-\omega_{d}^{2}+j 2 \zeta_{d} \omega_{c} \omega_{d}}\right| \\
& =\left|\frac{\left(\omega_{c}^{2}+j 2 \zeta_{d} \omega_{c} \omega_{d} \delta_{\zeta}\right)\left(\omega_{c}^{2}-\omega_{d}^{2}-j 2 \zeta_{d} \omega_{c} \omega_{d}\right)}{\left(\omega_{c}^{2}-\omega_{d}^{2}\right)^{2}+\left(2 \zeta_{d} \omega_{c} \omega_{d}\right)^{2}}\right| .
\end{aligned}
$$


Substituting $p_{0}=\omega_{c}^{2}-\omega_{d}^{2}$ and $p_{1}=\zeta_{d} \omega_{x} \omega_{d}$ and multiplying both sides by $p_{0}^{2}+4 p_{1}^{2}$, results in the following expressions:

$$
\begin{aligned}
R & =\frac{1}{\sqrt{2}}\left(p_{0}^{2}+4 p_{1}^{2}\right) \\
L & =\left|p_{0} \omega_{c}^{2}+4 p_{1}^{2} \delta_{\zeta}+j\left(2 p_{0} p_{1} \delta_{\zeta}-2 p_{1} \omega_{c}^{2}\right)\right| \\
& =\left[\left(p_{0} \omega_{c}^{2}+4 p_{1}^{2} \delta_{\zeta}\right)^{2}+\left(2 p_{0} p_{1} \delta_{\zeta}-2 p_{1} \omega_{c}^{2}\right)^{2}\right]^{\frac{1}{2}} \\
& =\left[p_{0}^{2} \omega_{c}^{4}+16 p_{1}^{4} \delta_{\zeta}^{2}+8 p_{0} p_{1}^{2} \omega_{c}^{2} \delta_{\zeta}\right. \\
& \left.+4 p_{0}^{2} p_{1}^{2} \delta_{\zeta}^{2}+4 p_{1}^{2} \omega_{c}^{4}-8 p_{0} p_{1}^{2} \omega_{c}^{2} \delta_{\zeta}\right]^{\frac{1}{2}} \\
& =\sqrt{\left(p_{0}^{2}+4 p_{1}^{2}\right)\left(\omega_{c}^{4}+4 p_{1}^{2} \delta_{\zeta}^{2}\right)} .
\end{aligned}
$$

In the following steps, the equation is simplified to express the bandwidth of the system $\omega_{d}$. The derivation closely follows the derivation for the bandwidth for a second order system without a zero in the numerator. Therefore, both sides are squared and multiplied by two:

$$
\begin{aligned}
& L=2\left(p_{0}^{2}+4 p_{1}^{2}\right)\left(\omega_{c}^{4}+4 p_{1}^{2} \delta_{\zeta}^{2}\right) \\
& R=\left(p_{0}^{2}+4 p_{1}^{2}\right)^{2} .
\end{aligned}
$$

Dividing both sides by $\left(p_{0}^{2}+4 p_{1}^{2}\right)$, re-substituting $p_{0}$ and $p_{1}$, and moving all terms containing $\omega_{d}$ to the right side results in:

$$
\begin{aligned}
& L=\omega_{c}^{4} \\
& R=\omega_{d}^{4}+2 \omega_{c}^{2} \omega_{d}\left(2 \zeta_{d}^{2}\left(1-2 \delta_{\zeta}^{2}\right)-1\right) .
\end{aligned}
$$

Substituting $p_{2}=2 \zeta_{d}^{2}\left(1-2 \delta_{\zeta}^{2}\right)-1$ and adding $\omega_{c}^{4} p_{2}^{2}$ on both sides results in another quadratic binomial equation on the right side:

$$
\begin{aligned}
& L=\omega_{c}^{4}\left(1+p_{3}^{2}\right) \\
& R=\omega_{d}^{4}+2 \omega_{c}^{2} \omega_{d}^{2} p_{2}+\omega_{c}^{4} p_{2}^{2}=\left(\omega_{d}^{2}+\omega_{c}^{2} p_{2}\right)^{2} .
\end{aligned}
$$

Taking the square root on both sides, but considering only the positive solutions and equating the left and right sides results in:

$$
\omega_{d}^{2}=\omega_{c}^{2}\left(\sqrt{1+p_{2}^{2}}-p_{2}\right) .
$$

Finally taking the square root again, but only considering the positive roots, and re-substituting $p_{2}$ leads to the adapted bandwidth formula as expressed in Eq. 13:

$$
\omega_{d}=\omega_{c} \sqrt{1-2 \zeta_{d}^{2}\left(1-2 \delta_{\zeta}^{2}\right)+\sqrt{1+\left(2 \zeta_{d}^{2}\left(1-2 \delta_{\zeta}^{2}\right)-1\right)^{2}}} .
$$

Note that when $\delta_{\zeta}=0$, when the transfer zero is ignored, we are indeed left with (9).

\section{APPENDIX B \\ UPPER BOUND DOB GAIN}

Based on the positive real condition, as shown in Eq. 16, an upper bound for the DOB gain $\alpha_{\max }$ can be found, such that the system is still passive. By using the following variation of the positive real condition:

$$
2 \operatorname{Re}\left(Z_{D O B}\right)=Z_{D O B}+Z_{D O B}^{*}>0,
$$

$$
Z_{D O B}(s)=k \frac{\left(s^{2}+2 \zeta_{q} \omega_{q} s+(1-\alpha) \omega_{q}^{2}\right)\left(s+2 \zeta_{n} \omega_{n}\right)}{\left(s^{2}+2 \zeta_{q} \omega_{q} s+\omega_{q}^{2}\right)\left(s^{2}+2 \zeta_{d} \omega_{c} s+\omega_{c}^{2}\right)},
$$

and solving it for $\alpha$, this maximum value can be found. Therefore, the following substitutions are defined: $\hat{\omega}=\frac{\omega}{\omega_{q}}$, $\tilde{\omega}=\frac{\omega}{\omega_{c}}$, and $\delta=\frac{\zeta_{n} \omega_{n}}{\zeta_{d} \omega_{c}}$. Here, $\zeta_{q}$ is the damping ratio of DOB filter $Q$, which is $\zeta_{q}=\frac{1}{\sqrt{2}}$ for a Butterworth filter, or higher for reasonable filters. Inserting those into Eq. 28 and solving Eq. 27 results in an upper bound of:

$$
\begin{aligned}
& \alpha_{\max }=\min _{\omega} \alpha(\omega), \forall \omega: \alpha(\omega)>0 \\
& \alpha(\omega)=\frac{\left(1+\hat{\omega}^{4}+2 \hat{\omega}^{2}\left(2 \zeta_{q}^{2}-1\right)\right)\left(\delta+\tilde{\omega}^{2}(1-\delta)\right)}{\left(1+\hat{\omega}^{2}\right)\left(\delta+\tilde{\omega}^{2}(1-\delta)\right)+4 \zeta_{q} \zeta_{d} \tilde{\omega} \hat{\omega}\left(\frac{1-\tilde{\omega}^{2}}{4 \zeta_{d}^{2}}-\delta\right)},
\end{aligned}
$$

All other conditions result in a lower bound that is below zero. Based on the limitation for negative feedback, these cases can be eliminated. Furthermore, the result for negative conditions also results in a lower bound. In general, the maximum value for the DOB gain that still guarantees passivity is located at a low frequency and is found by numerically minimizing the right hand side of Eq. 29 at those frequencies.

\section{REFERENCES}

[1] C. Meijneke, W. van Dijk, and H. van der Kooij, "Achilles: an autonomous lightweight ankle exoskeleton to provide push-off power," in Biomedical Robotics and Biomechatronics (2014 5th IEEE RAS \& EMBS International Conference on. IEEE, 2014, pp. 918-923.

[2] K. A. Witte, J. Zhang, R. W. Jackson, and S. H. Collins, "Design of two lightweight, high-bandwidth torque-controlled ankle exoskeletons," in Robotics and Automation (ICRA), 2015 IEEE International Conference on. IEEE, 2015, pp. 1223-1228.

[3] C. B. Sanz-Morère, M. Fantozzi, A. Parri, F. Giovacchini, A. Baldoni, S. Crea, and N. Vitiello, "A bioinspired control strategy for the cyberlegs knee-ankle-foot orthosis: Feasibility study with lower-limb amputees," in 2018 7th IEEE International Conference on Biomedical Robotics and Biomechatronics (Biorob). IEEE, 2018, pp. 503-508.

[4] S. Wang, L. Wang, C. Meijneke, E. Van Asseldonk, T. Hoellinger, G. Cheron, Y. Ivanenko, V. La Scaleia, F. Sylos-Labini, M. Molinari et al., "Design and control of the mindwalker exoskeleton," IEEE transactions on neural systems and rehabilitation engineering, vol. 23, no. 2, pp. 277-286, 2015.

[5] N. A. Radford, P. Strawser, K. Hambuchen, J. S. Mehling, W. K. Verdeyen, A. S. Donnan, J. Holley, J. Sanchez, V. Nguyen, L. Bridgwater et al., "Valkyrie: Nasa's first bipedal humanoid robot," Journal of Field Robotics, vol. 32, no. 3, pp. 397-419, 2015.

[6] M. A. Hopkins, S. A. Ressler, D. F. Lahr, A. Leonessa, and D. W. Hong, "Embedded joint-space control of a series elastic humanoid," in Intelligent Robots and Systems (IROS), 2015 IEEE/RSJ International Conference on. IEEE, 2015, pp. 3358-3365.

[7] S. K. Au, J. Weber, and H. Herr, "Powered ankle-foot prosthesis improves walking metabolic economy," IEEE Transactions on Robotics, vol. 25, no. 1, pp. 51-66, 2009.

[8] J. K. Hitt, T. G. Sugar, M. Holgate, and R. Bellman, "An active footankle prosthesis with biomechanical energy regeneration," Journal of medical devices, vol. 4, no. 1, p. 011003, 2010.

[9] J. M. Caputo and S. H. Collins, "A universal ankle-foot prosthesis emulator for human locomotion experiments," Journal of biomechanical engineering, vol. 136, no. 3, p. 035002, 2014.

[10] A. F. Azocar, L. M. Mooney, L. J. Hargrove, and E. J. Rouse, "Design and characterization of an open-source robotic leg prosthesis," in 2018 7th IEEE International Conference on Biomedical Robotics and Biomechatronics (Biorob). IEEE, 2018, pp. 111-118.

[11] C. Meijneke, S. Wang, V. Sluiter, and H. van der Kooij, "Introducing a modular, personalized exoskeleton for ankle and knee support of individuals with a spinal cord injury," in Wearable Robotics: Challenges and Trends. Springer, 2017, pp. 169-173. 
[12] N. Paine, J. S. Mehling, J. Holley, N. A. Radford, G. Johnson, C.-L. Fok, and L. Sentis, "Actuator control for the nasa-jsc valkyrie humanoid robot: A decoupled dynamics approach for torque control of series elastic robots," Journal of Field Robotics, vol. 32, no. 3, pp. 378-396, 2015.

[13] N. G. Tsagarakis, M. Laffranchi, B. Vanderborght, and D. G. Caldwell, "A compact soft actuator unit for small scale human friendly robots," in Robotics and Automation, 2009. ICRA'09. IEEE International Conference on. IEEE, 2009, pp. 4356-4362.

[14] K. Kong, H. Moon, B. Hwang, D. Jeon, and M. Tomizuka, "Impedance compensation of subar for back-drivable force-mode actuation," IEEE Transactions on Robotics, vol. 25, no. 3, pp. 512-521, 2009.

[15] H. Vallery, J. Veneman, E. Van Asseldonk, R. Ekkelenkamp, M. Buss, and H. Van Der Kooij, "Compliant actuation of rehabilitation robots," IEEE Robotics \& Automation Magazine, vol. 15, no. 3, 2008.

[16] K. Kong, J. Bae, and M. Tomizuka, "Control of rotary series elastic actuator for ideal force-mode actuation in human-robot interaction applications," IEEE/ASME transactions on mechatronics, vol. 14, no. 1, pp. 105-118, 2009.

[17] N. Paine, S. Oh, and L. Sentis, "Design and control considerations for high-performance series elastic actuators," IEEE/ASME Transactions on Mechatronics, vol. 19, no. 3, pp. 1080-1091, 2014.

[18] G. Wyeth, "Control issues for velocity sourced series elastic actuators," in Proceedings of the Australasian Conference on Robotics and Automation 2006. Australian Robotics and Automation Association Inc, 2006.

[19] H. Vallery, R. Ekkelenkamp, H. Van Der Kooij, and M. Buss, "Passive and accurate torque control of series elastic actuators," in Intelligent Robots and Systems, 2007. IROS 2007. IEEE/RSJ International Conference on. IEEE, 2007, pp. 3534-3538.

[20] G. A. Pratt, P. Willisson, C. Bolton, and A. Hofman, "Late motor processing in low-impedance robots: Impedance control of series-elastic actuators," in American Control Conference, 2004. Proceedings of the 2004, vol. 4. IEEE, 2004, pp. 3245-3251.

[21] A. Calanca, R. Muradore, and P. Fiorini, "A review of algorithms for compliant control of stiff and fixed-compliance robots," IEEE/ASME Transactions on Mechatronics, vol. 21, no. 2, pp. 613-624, 2016.

[22] A. Q. Keemink, H. van der Kooij, and A. H. Stienen, "Admittance control for physical human-robot interaction," The International Journal of Robotics Research, p. 0278364918768950, 2017.

[23] S. Oh and K. Kong, "High-precision robust force control of a series elastic actuator," IEEE/ASME Transactions on Mechatronics, vol. 22, no. 1 , pp. 71-80, 2017.

[24] G. A. Pratt and M. M. Williamson, "Series elastic actuators," in Intelligent Robots and Systems 95.'Human Robot Interaction and Cooperative Robots', Proceedings. 1995 IEEE/RSJ International Conference on, vol. 1. IEEE, 1995, pp. 399-406.

[25] A. Calanca and P. Fiorini, "A rationale for acceleration feedback in force control of series elastic actuators," IEEE Transactions on Robotics, vol. 34 , no. 1 , pp. 48-61, 2018.

[26] _ - "Human-adaptive control of series elastic actuators," Robotica, vol. 32, no. 8, pp. 1301-1316, 2014

[27] D. P. Losey, A. Erwin, C. G. McDonald, F. Sergi, and M. K. OMalley, "A time-domain approach to control of series elastic actuators: Adaptive torque and passivity-based impedance control," IEEE/ASME Transactions on Mechatronics, vol. 21, no. 4, pp. 2085-2096, 2016.

[28] J. Zhang, C. C. Cheah, and S. H. Collins, "Experimental comparison of torque control methods on an ankle exoskeleton during human walking," in Robotics and Automation (ICRA), 2015 IEEE International Conference on. IEEE, 2015, pp. 5584-5589.

[29] E. Schrijver and J. Van Dijk, "Disturbance observers for rigid mechanical systems: Equivalence, stability, and design," Journal of Dynamic Systems, Measurement, and Control, vol. 124, no. 4, pp. 539-548, 2002.

[30] K. Kong and M. Tomizuka, "Nominal model manipulation for enhancement of stability robustness for disturbance observer-based control systems," International Journal of Control, Automation and Systems, vol. 11, no. 1, pp. 12-20, 2013.

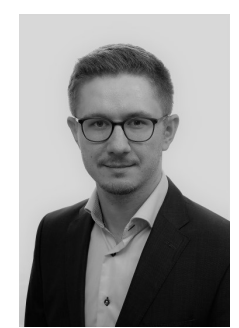

Wolfgang F. Rampeltshammer is a $\mathrm{PhD}$ student at the Department of Biomechanical Engineering at the University of Twente, the Netherlands. He received his MSc in Electrical Engineering and Information Technology with high distinction in 2016 from the Technical University Munich, Germany. His research focus are in the control of lower limb exosekeletons to augment capabilities of healthy users by utilizing human-in-the-loop methods and to restore gait of patients with paraplegia.

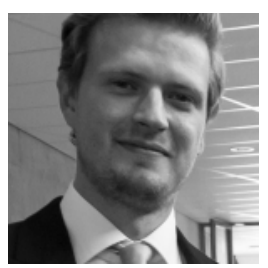

Arvid Q.L. Keemink is Assistant Professor in Biorobotics at the Department of Biomechanical Engineering at the University of Twente, The Netherlands. He obtained his MSc in Mechatronics with honors (cum laude) in 2012 and received his $\mathrm{PhD}$ in 2017. His main interests are in control of biorobotics, especially on the development of safe low-level physical interaction and on humaninspired, optimal and self-learning high level control behavior to complement or augment deficient human motor performance with assistive devices.

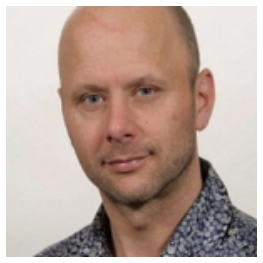

Herman van der Kooij, received his $\mathrm{PhD}$ with honors (cum laude) in 2000 and is from 2010 full professor in Biomechatronics and Rehabilitation Technology at the Department of Biomechanical Engineering at the University of Twente and Delft University of Technology, the Netherlands. His expertise and interests are in the field of human motor control, adaptation, and learning, rehabilitation robots, diagnostic, and assistive robotics, virtual reality, rehabilitation medicine, and neuro-computational modeling. 